\title{
A CHARACTERIZATION OF SEMI-PRIME IDEALS IN NEAR-RINGS
}

\author{
N. J. GROENEWALD
}

(Received 21 June 1982)

Communicated by R. Lidl

\begin{abstract}
It is well-known that in any near-ring, any intersection of prime ideals is a semi-prime ideal. The aim of this note is to prove that any ideal is a prime ideal if and only if it is equal to its prime radical. As a consequence of this we have any semi-prime ideal $I$ in a near-ring $N$ is the intersection of minimal prime ideals of $I$ in $N$ and that $I$ is the intersection of all prime ideals containing $I$.
\end{abstract}

1980 Mathematics subject classification (Amer. Math. Soc.): primary 16 A 76; secondary 08 A 40.

\section{Preliminaries}

A near-ring is an algebraic system, $(N,+, \cdot)$ satisfying (i) $(N,+)$ is a group, (ii) $(N, \cdot)$ is a semi-group and (iii) $(x+y) z=x z+y z$ for all $x, y, z$ in $N$. We abbreviate $(N,+, \cdot)$ by $N$.

If $S$ and $T$ are subsets of $N$, we denote the set $\{s t: s \in S, t \in T\}$ by $S T$. A normal subgroup $I$ of $(N,+)$ is called an ideal of $N(I \unlhd N)$ if $I N \subset I$ and for all $n, n^{\prime} \in N$ and all $i \in I, n\left(n^{\prime}+i\right)-n n^{\prime} \in I$. An ideal $P$ of $N$ is called a prime ideal if for any ideal $I$ and $J$ of $N, I J \subseteq P$ implies either $I \subseteq P$ or $J \subseteq P$. An ideal $I$ of $N$ is called a semi-prime ideal if for any ideal $J$ of $N, J^{2} \subseteq I$ implies that $J \subseteq I$. An ideal minimal in the set of all prime ideals containing some given ideal $I$ is called a minimal prime ideal of $I$ in $N$.

If $x$ is an element of $N$, then the principal ideal generated by $x$ is denoted by (x). If $S$ is a subset of $N$, we write $N-S=\{n \in N / n \notin S\}$. A subset $M$ of a near-ring $N$ is called an $m$-system if for any $a, b \in M$ there exist $a_{1} \in(a)$ and

(c) 1983 Australian Mathematical Society $0263-6115 / 83 \$ A 2.00+0.00$ 
$b_{1} \in(b)$ such that $a_{1} b_{1} \in M$. A subset $S$ of a near-ring $N$ is called an $S p$-system if for any $s \in S$, there exists $s_{1} \in(s)$ and $s_{1} \in(s)$ such that $s_{1} s_{2} \in S$. The prime radical $\mathscr{P}(I)$ of the ideal $I$ consists of those elements $n \in N$ with the property that every $m$-system which contains $n$, contains an element of $I$.

In this section we prove the main theorem. Before proving this, we state the following result.

LEMMA 2.1 (Van der Walt [3]). If $I$ is an ideal in the near-ring $N$ then the prime radical $\mathcal{P}(I)$ of the ideal $I$ is the intersection of all the minimal prime ideals of $I$ in $N$.

THEOREM 2.2. An ideal $I$ in a near-ring $N$ is a semi-prime ideal in $N$ if and only if $\mathscr{P}(I)=I$.

Proof. The "if" part is an immediate consequence of Theorem 3 of Van der Walt [3] and the fact that the intersection of prime ideals is a semi-prime ideal. To prove the "only if" part, suppose that $I$ is a semi-prime ideal in $N$. Certainly, $I \subseteq \mathscr{P}(I)$, so let us assume $I \subset \mathscr{P}(I)$ and seek a contradiction. Suppose $a \in \mathscr{P}(I)$ with $a \notin I$. Hence $N-I$ is an $S p$-system. So by 2.92 of Pilz [1], there exists an $m$-system $M$ in $N$ such that $a \in M \subseteq N-I$. Now $a \in \mathscr{P}(I)$ and, by the definition of $\mathscr{P}(I)$, every $m$-system which contains $a$ meets $I$. But $I \cap(N-I)$ is empty, and therefore $M \cap I$ is empty. This gives the desired contradiction and completes the proof of the theorem.

As corollaries, we get the following results proved by Sambasiva Rao [2].

COROllary 2.3. If I is any semi-prime ideal in the near-ring $N$, then I is the intersection of all minimal prime ideals of I in $N$.

PRoof. Immediate from Lemma 2.1 and Theorem 2.2.

COROLlaRY 2.4. If $I$ is a semi-prime ideal in a near-ring $N$, then $I$ is the intersection of all prime ideals containing $I$.

Proof. Immediate from Theorem 2.2 and Van der Walt [3], Theorem 3. 


\section{References}

[1] G. Pilz (1977), Near-rings (North-Holland, Amsterdam-New York).

[2] V. Sambasiva Rao, 'A characterization of semi-prime ideals in near-rings', J. Austral. Math. Soc. Ser. A 32 (1982), 212-214.

[3] A. P. J. Van der Walt, 'Prime ideals and nil radicals in near-rings', Arch. Math. (Basel) 15 (1964), 408-414.

Department of Mathematics

University of Port Elizabeth

6000 Port Elizabeth

South Africa 